\title{
Metabolismo de cálcio em ovinos em crescimento sob suplementação com diferentes fontes de cálcio: aplicação e comparação de dois modelos matemáticos ${ }^{1}$
}

\section{Dorinha Miriam Silber Schmidt Vitti ${ }^{2}$, Ana Paula Roque ${ }^{3}$, Raquel Souza Dias ${ }^{4}$, João Batista Lopes $^{4}$, Ives Cláudio da Silva Bueno ${ }^{5}$, Mauro Sartori Bueno ${ }^{6}$, Eduardo Fernando Nozella ${ }^{4}$}

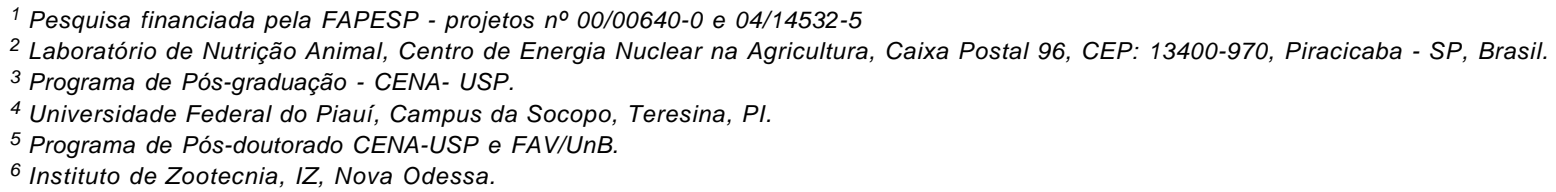

RESUMO - Este trabalho foi realizado com o objetivo de estudar, por meio de dois modelos matemáticos, o metabolismo do cálcio (Ca) em ovinos recebendo dietas suplementadas com diferentes fontes de Ca. Foram utilizados 20 cordeiros mestiços Santa Inês ( 6 a 8 meses de idade) alimentados com uma dieta basal composta de milho, farelo de soja, bagaço de cana hidrolisado, uréia, fosfato monoamônio (MAP) e mistura mineral suplementada com cinco fontes de cálcio: polpa cítrica (PC), feno de alfafa (FA), calcário calcítico (CC), farinha de conchas de ostras (FC) e fosfato bicálcico (FB). Os animais receberam, via jugular, solução contendo 7,7 MBq de ${ }^{45} \mathrm{Ca}$. Durante sete dias, realizaram-se coletas de fezes, sangue e urina. Após o período de coleta, os animais foram sacrificados para retirada de amostras de tecidos (fígado, rins, coração, músculo e 12a costela) para análises. O metabolismo de Ca foi avaliado pelos modelos de Vitti et al. (2000) e Fernandez (1995). O delineamento experimental foi o inteiramente casualizado e as médias foram comparadas pelo teste Duncan. Não houve diferença significativa para o consumo de Ca entre os tratamentos. A excreção fecal foi menor para o CC. O balanço de Ca foi negativo para FA e PC. A absorção de Ca pode ter sido prejudicada pela presença de pectina e oxalato na polpa cítrica e no feno de alfafa, respectivamente. Os fluxos entre o compartimento central e o trato digestivo indicaram maior absorção de Ca para o CC. As fontes CC, FB e FC apresentaram maior disponibilidade cálcio, com valores respectivos de 65,38; 34,12 e 32,43\%. A comparação entre os modelos comprovou diferenças nos fluxos entre o sangue e os tecidos e ossos, todavia, o balanço entre esses compartimentos foi similar. As diferenças entre os modelos possivelmente foram ocasionadas pela sua estrutura.

Palavras-chave: metabolismo, modelo, ovinos, radio-cálcio

\section{Calcium metabolism in growing sheep supplemented with different calcium sources: application and comparison of two mathematical models}

\begin{abstract}
This research was conducted to compare the accuracy of two mathematical models to predict calcium (Ca) metabolism in sheep supplemented with different Ca sources. Twenty Brazilian male sheep averaging 7 to 8 months of age were fed a basal diet containing corn, soybean meal, hydrolyzed sugarcane bagasse, urea, mono-ammonium phosphate, mineral mixture and one of the following Ca sources: limestone (LM), alfalfa hay (AH), dicalcium phosphate (DP), oyster shell meal $(\mathrm{SM})$, and citrus pulp (CP). On day 22 each animal received an intravenous injection of $7.7 \mathrm{MBq}$ of radio-calcium $\left({ }^{45} \mathrm{Ca}\right)$ followed by collection of blood, feces, and urine every $24 \mathrm{~h}$ for 7 days. Total $\mathrm{Ca}$ and radioactivity were measured in all samples. At the end of the collection period, animals were slaughtered and samples of liver, heart, kidney, muscles and $12^{\text {th }}$ rib were taken for analysis. Calcium metabolism was evaluated by the models proposed by Vitti et al. (2000) and Fernandez (1995). A completely randomized design was used and means were compared by the Duncan test. No significant differences were observed for $\mathrm{Ca}$ intake among treatments. However, total fecal excretion of Ca differed and was lowest in animals fed LM. Calcium balance was negative in sheep receiving $\mathrm{AH}$ and $\mathrm{CP}$ because $\mathrm{Ca}$ absorption might have been compromised by the high contents of pectin and oxalate in these two supplements, respectively. Flows of Ca between the central pool and the digestive tract showed the greatest absorption of Ca on animals fed LM. Diets supplemented with LM, DP, and SM had highest Ca availability with values of $65.38,34.12$ and $32.43 \%$, respectively. The models differed in the predictions of Ca flows among blood, bone and soft tissues but showed similar values for Ca balance in the different compartments. These discrepant results may be explained by structural difference between models.
\end{abstract}

Key Words: metabolism, model, radio-calcium, sheep 


\section{Introdução}

$\mathrm{O}$ cálcio $(\mathrm{Ca})$ é considerado um dos minerais mais importantes na produção de ruminantes, pois desempenha inúmeras funções básicas relacionadas à integridade do esqueleto, à manutenção da permeabilidade normal das células, à coagulação do sangue e à regulação da excitabilidade neuromuscular (Andriguetto et al., 1993).

$\mathrm{Na}$ formulação de dietas para ruminantes, o cálcio consiste em um importante nutriente e pode ser originado tanto de produtos inorgânicos como de ingredientes de origem vegetal. Alguns alimentos, por possuírem elevado teor de cálcio em sua composição, podem funcionar como fonte desse mineral, desde que sua disponibilidade seja conhecida.

Entre as fontes de cálcio, a polpa cítrica destaca-se por ser um subproduto da indústria de suco de laranja (o Brasil é o maior produtor mundial), o que garante a oferta desse ingrediente no mercado nacional (Mejía \& Ferreira, 2000). De acordo com o NRC (1985), o calcário calcítico, a farinha de conchas de ostras e o fosfato bicálcico são os principais suplementos de Ca utilizados na alimentação animal (Peixoto \& Maier, 1993).

Quanto ao metabolismo do cálcio, os estudos físicoquímicos comprovam que as trocas de cálcio entre os ossos e fluidos corporais ocorrem por dois processos: a) trocas iônicas, que correspondem ao processo rápido, na superfície óssea, quando o excesso de cálcio é incorporado à molécula de fosfato tricálcico; b) trocas lentas ou processos de recristalização, que correspondem à penetração de cálcio trocável no interior do osso (Aubert \& Milhaud,1960).

Uma variedade de técnicas tem sido utilizada no estudo do metabolismo de minerais em animais, incluindo o balanço convencional e o uso de radioisótopos. Traçadores radioativos têm sido utilizados no estudo do metabolismo e da distribuição dos minerais, especialmente do fósforo $(\mathrm{P})$, por meio de modelos matemáticos envolvendo o fluxo entre os diversos compartimentos do organismo animal (Grace, 1981; Schneider et al., 1985; Vitti et al., 2000; Kebreab et al., 2004; Vitti et al., 2005) e análises por programas de computadores (Boston et al., 1981). Pfeffer et al. (2005) descreveram, com detalhes, alguns dos modelos e enfatizaram sua aplicação para estudos do impacto ambiental da excreção de $\mathrm{P}$ pelos animais domésticos.

Uma análise quantitativa do metabolismo de $\mathrm{P}$ nos animais domésticos foi feita por Bravo et al. (2003), que utilizaram dados compilados de mais de cem publicações referentes ao metabolismo de P. Esses autores aplicaram uma série de equações e modelos matemáticos para descrever os aspectos quantitativos da absorção e excreção de $P$.
Outros trabalhos com P têm sido realizados com uso de radioisótopos (Challa et al., 1989; Vitti et al., 1992, Salviano \& Vitti, 1998; Vitti et al., 2005), mas são poucas as informações sobre o uso de cálcio. Vitti et al. (2000) construíram um modelo matemático sobre o fluxo do fósforo em compartimentos fisiológicos e anatômicos de caprinos usando dados obtidos pela técnica de diluição isotópica. Neste contexto, Fernandez (1995), utilizando dados combinados de balanço e cinética de radioisótopos, formulou e resolveu um modelo de metabolismo de $\mathrm{Pe}$ Ca para avaliar os efeitos de níveis crescentes desses minerais em dietas para suínos. Os estudos mais recentes sobre o metabolismo de Ca são desenvolvidos com humanos, especialmente para o estudo da cinética nos ossos (Beck et al., 2003).

Este trabalho foi desenvolvido com a finalidade de estudar o metabolismo do cálcio e comparar os resultados obtidos em dois modelos matemáticos destinados à avaliação das trocas de Ca entre os compartimentos fisiológicos ou anatômicos de ovinos alimentados com dietas suplementadas com diferentes fontes de $\mathrm{Ca}$.

\section{Material e Métodos}

A pesquisa foi desenvolvida no Laboratório de Nutrição Animal do Centro de Energia Nuclear na Agricultura da Universidade de São Paulo. Foram selecionados 20 cordeiros mestiços Santa Inês (machos não-castrados) com 7 a 8 meses de idade. Esses animais foram tosquiados e receberam vermífugo antes do período experimental.

Os tratamentos consistiram de uma dieta basal composta de milho, farelo de soja, bagaço de cana hidrolisado, uréia, fosfato monoamônio (MAP) e mistura mineral, sendo suplementada com cinco diferentes fontes de cálcio: polpa cítrica (PC), feno de alfafa (FA), calcário calcítico (CC), farinha de conchas de ostras (FC) e fosfato bicálcico (FB).

As dietas foram formuladas com base nas exigências preconizadas pelo NRC (1985) para cordeiros em terminação com ganho de peso médio diário de $295 \mathrm{~g}$, considerando consumo de 4,3\% do peso vivo do animal. A composição e a caracterização da dieta são apresentadas na Tabela 1.

Os animais foram alojados em gaiolas para estudos de metabolismo por um período de 28 dias. No $22 \underline{0}$ dia experimental, através da jugular direita, foram injetados em cada animal 7,7 Mbq de ${ }^{45} \mathrm{Ca}$ (em $1 \mathrm{~mL}$ de solução salina $0,85 \%$ ). Foram coletadas amostras de sangue pela jugular esquerda, utilizando-se tubos a vácuo contendo heparina. Os períodos de coleta foram cinco minutos após a injeção e depois a cada 24 horas, durante sete dias. As coletas totais de fezes e urina foram feitas diariamente, 24 horas após a injeção do radioisótopo, até o 8음ia. Alíquotas correspondentes a 1/10 
Tabela 1 - Composição das dietas experimentais (100\% MS) Table 1 - Composition of the experimental diets (\%DM)

\begin{tabular}{|c|c|c|c|c|c|}
\hline \multirow{2}{*}{$\begin{array}{l}\text { Composição } \\
\text { Composition } \\
\text { Ingrediente } \\
\text { Ingredient }\end{array}$} & \multicolumn{5}{|c|}{$\begin{array}{c}\text { Dieta experimental } \\
\text { Experimental diet }\end{array}$} \\
\hline & $\begin{array}{l}C C \\
L M\end{array}$ & $\begin{array}{l}\text { FA } \\
A H\end{array}$ & $\begin{array}{l}\mathrm{PC} \\
C P\end{array}$ & $\begin{array}{l}\mathrm{FB} \\
D P\end{array}$ & $\begin{array}{l}\text { FC } \\
S M\end{array}$ \\
\hline Polpa cítrica & - & - & 30,0 & - & - \\
\hline $\begin{array}{l}\text { Citrus pulp } \\
\text { Feno de alfafa }\end{array}$ & - & 45,0 & - & - & - \\
\hline $\begin{array}{l}\text { Alfalfa hay } \\
\text { Calcário calcítico } \\
\text { Limestone }\end{array}$ & 1,3 & - & - & - & - \\
\hline $\begin{array}{l}\text { Farinha de conchas } \\
\text { de ostras } \\
\text { Oyster shell meal }\end{array}$ & - & - & - & - & 1,2 \\
\hline Fosfato bicálcico & - & - & - & 2,0 & - \\
\hline $\begin{array}{l}\text { Dicalcium phosphate } \\
\text { Milho }\end{array}$ & 40,0 & 40,0 & 14,0 & 39,5 & 40,0 \\
\hline $\begin{array}{l}\text { Corn } \\
\text { Bagaço de cana } \\
\text { Sugarcane bagasse }\end{array}$ & 42,0 & 13,8 & 39,4 & 43,3 & 43,1 \\
\hline $\begin{array}{l}\text { Farelo de soja } \\
\text { Soybean meal }\end{array}$ & 15,0 & - & 15,0 & 14,0 & 14,0 \\
\hline Uréia & 0,7 & 0,5 & 0,6 & 0,7 & 0,7 \\
\hline $\begin{array}{l}\text { Urea } \\
\text { MAP }^{1} \\
\text { Mistura mineral }\end{array}$ & 0,5 & $\begin{array}{l}0,2 \\
0.5\end{array}$ & $\begin{array}{l}0,5 \\
0.5\end{array}$ & $0 . \overline{5}$ & $\begin{array}{l}0,5 \\
0,5\end{array}$ \\
\hline $\begin{array}{l}\text { Mineral mixture } \\
\mathrm{MS}(\%) \\
D M\end{array}$ & 81,96 & 83,66 & 83,38 & 81,78 & 81,29 \\
\hline Cinzas (\%) & 6,53 & 5,41 & 6,20 & 6,79 & 5,94 \\
\hline FDN (\%) & 34,82 & 33,77 & 35,72 & 35,30 & 35,27 \\
\hline $\begin{array}{l}N D F \\
\text { FDA }(\%) \\
A D F\end{array}$ & 27,49 & 23,60 & 29,29 & 28,04 & 27,97 \\
\hline $\begin{array}{l}\mathrm{Ca}(\%) \\
\mathrm{PB}(\%)\end{array}$ & $\begin{array}{r}0,59 \\
13,49\end{array}$ & $\begin{array}{r}0,54 \\
15,54\end{array}$ & $\begin{array}{r}0,53 \\
13,90\end{array}$ & $\begin{array}{r}0,63 \\
13,41\end{array}$ & $\begin{array}{r}0,58 \\
13,37\end{array}$ \\
\hline $\mathrm{CP}$ & & & & & \\
\hline $\mathrm{P}(\%)$ & 0,36 & 0,33 & 0,33 & 0,60 & 0,35 \\
\hline
\end{tabular}

do total diário excretado foram coletadas e congeladas para as análises.

Após a coleta, o sangue foi centrifugado $(1.100 \mathrm{~g})$ por dez minutos para separação do plasma e do sobrenadante, usado para análise do teor de Ca por espectrofotometria de absorção atômica (Zagatto et al., 1979).

As amostras de fezes foram maceradas, homogeneizadas e, após digestão das cinzas com ácido clorídrico concentrado, efetuou-se a leitura do $\mathrm{Ca}$ por espectrofotometria de absorção atômica (Zagatto et al., 1979). O teor de Ca inorgânico na urina foi determinado segundo Morse et al. (1992).
Para detecção de ${ }^{45} \mathrm{Ca}$, preparou-se uma solução cintiladora contendo $4 \mathrm{~g}$ de difeniloxazole (PPO) e $0,3 \mathrm{~g}$ de 1,4 bis-(5-feniloxazole-2-il) benzeno (POPOP) dissolvidos em mistura de $500 \mathrm{~mL}$ de triton $\mathrm{X}-100$ e $1 \mathrm{~L}$ de tolueno.

As amostras de plasma e urina foram descongeladas e homogeneizadas. Pipetou-se $1 \mathrm{~mL}$ da amostra acidificada com três gotas de $\mathrm{HCl} 2 \mathrm{~N}$ e $10 \mathrm{~mL}$ de solução cintiladora em frascos de borosilicato para cintilação líquida. As amostras de fezes foram descongeladas, maceradas, homogeneizadas e, após digestão das cinzas com $\mathrm{HCl} 2 \mathrm{~N}$, $1 \mathrm{~mL}$ desta solução foi adicionado a $10 \mathrm{~mL}$ de solução cintiladora em frascos de borosilicato para medição da radioatividade por cintilação líquida.

Após o período de coleta, os cordeiros foram abatidos conforme orientação da Comissão de Ética em Experimentação com Animais do CENA. Foram coletadas amostras de tecidos (12a costela, fígado, rim, coração músculo) para a determinação dos teores de Ca inorgânico e radioativo. As carcaças foram posteriormente levadas para um depósito construído de acordo com normas da Comissão Nacional de Energia Nuclear (CNEN). Todas as operações com radioisótopos foram supervisionadas pelo Serviço de Proteção Radiológica do CENA.

O delineamento experimental utilizado foi o inteiramente casualizado, com cinco tratamentos e quatro repetições e as médias foram comparadas pelo teste Duncan (SAS, 2000).

O modelo matemático 1 , do fluxo de cálcio nos compartimentos fisiológicos e anatômicos do organismo de ovinos, seguiu as recomendações de Vitti et al. (2000), em trabalho sobre cinética com radioisótopos desenvolvidos em caprinos (Figura 1).

$\mathrm{Na}$ estruturação do modelo, foram considerados quatro compartimentos: 1 . trato digestivo; 2 . sangue; 3 . ossos; e 4. tecidos moles. O fluxo de Ca entre os compartimentos, dentro e fora do sistema, foi caracterizado pelas setas. O trato digestivo, o osso e os tecidos moles estiveram em intercâmbio bi-direcional com opool do sangue, de acordo com os fluxos $\mathrm{F}_{21}$ e $\mathrm{F}_{12}, \mathrm{~F}_{23}$ e $\mathrm{F}_{32}$ e $\mathrm{F}_{24}$ e $\mathrm{F}_{42}$, respectivamente.

A entrada de Ca no sistema ocorre via ingestão $\left(\mathrm{F}_{10}\right) \mathrm{e}$ a saída, por meio das fezes $\left(\mathrm{F}_{01}\right)$ e da urina $\left(\mathrm{F}_{02}\right)$. O cálcio marcado ${ }^{45} \mathrm{Ca}$ foi administrado como uma única dose, $\mathrm{D}$ cpm, para o tempo zero, sendo o valor e a atividade específica nos pools de sangue, no osso e nos tecidos moles medidos após oito dias da introdução. Nesse esquema, assumiu-se que não houve reentrada de $\mathrm{Ca}$ marcado a partir de fontes externas.

O princípio da conservação das massas pode ser aplicado em cada pool na Figura 1 para gerar as equações diferenciais, que descrevem o comportamento dinâmico do 


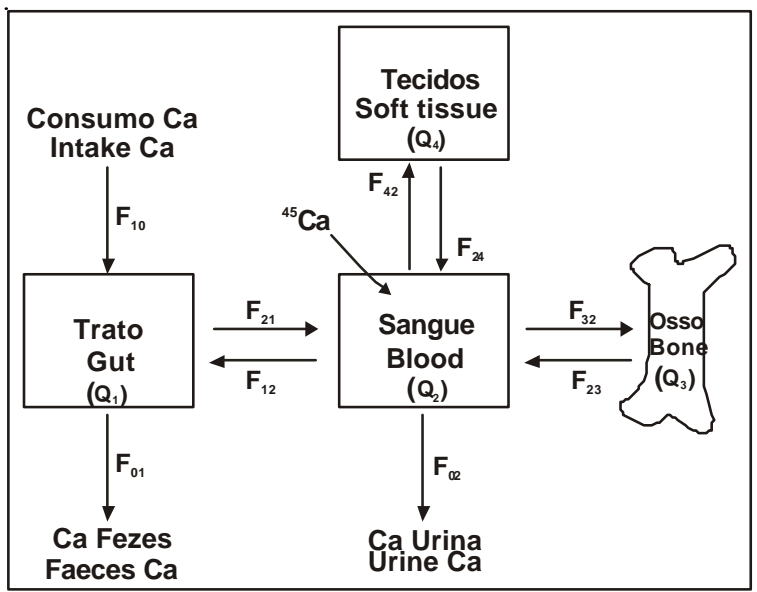

Figura 1 - Esquema do modelo de metabolismo de cálcio de acordo com Vitti et al. (2000).

Figure 1 - Metabolism of Ca according to Vitti et al. (2000) model.

sistema. Assim, as equações diferenciais de 01 a 04 representam esse princípio para o Ca não-marcado e as equações diferenciais de 05 a 08 para o $\mathrm{Ca}$ marcado, conforme notação definida abaixo.

Os principais símbolos usados no desenvolvimento do modelo são: Fij = fluxo total de Ca para o pool i a partir do j; $\mathrm{Fi} 0$ = fluxo externo dentro do pool $\mathrm{i} ;$ e F0j = fluxo de saída do sistema a partir do pool $\mathrm{j}$, o sublinhado indica o fluxo que pode ser medido experimentalmente: $\mathrm{g} / \mathrm{d} ; \mathrm{D}=$ dose de ${ }^{45} \mathrm{Ca}$ administrada no sangue no tempo zero: cpm; $\mathrm{Qi}=$ quantidade total de Ca no pool i: $\mathrm{g}$; qi = quantidade de ${ }^{45} \mathrm{Ca}$ no pool i: $\mathrm{cpm}$; si = atividade específica do pool $\mathrm{i}(=\mathrm{qi} / \mathrm{Qi}): \mathrm{cpm} / \mathrm{g} ; \mathrm{T}=$ tempo, em dias.

$\mathrm{dQ}_{1} / \mathrm{dt}=\mathrm{F}_{10}+\mathrm{F}_{12}-\mathrm{F}_{01}-\mathrm{F}_{21}$

$\mathrm{dQ}_{2} / \mathrm{dt}=\mathrm{F}_{21}+\mathrm{F}_{23}+\mathrm{F}_{02}-\mathrm{F}_{12}-\mathrm{F}_{32}-\mathrm{F}_{42}$

$\mathrm{dQ}_{3} / \mathrm{dt}=\mathrm{F}_{32}-\mathrm{F}_{23}$

$\mathrm{dQ}_{4} / \mathrm{dt}=\mathrm{F}_{42}-\mathrm{F}_{24}$

$\mathrm{dq}_{1} / \mathrm{dt}=\mathrm{s}_{2} \mathrm{~F}_{12}-\mathrm{s}_{1}\left(\mathrm{~F}_{01}+\mathrm{F}_{21}\right)$

$\mathrm{dq}_{2} / \mathrm{dt}=\mathrm{s}_{1} \mathrm{~F}_{21}+\mathrm{s}_{3} \mathrm{~F}_{23}+\mathrm{s}_{4} \mathrm{~F}_{24}-\mathrm{s}_{2}\left(\mathrm{~F}_{02}+\mathrm{F}_{12}+\mathrm{F}_{32}+\mathrm{F}_{42}\right)$

$\mathrm{dq}_{3} / \mathrm{dt}=\mathrm{s}_{2} \mathrm{~F}_{32}-\mathrm{s}_{3} \mathrm{~F}_{23}$

$\mathrm{dq}_{4} / \mathrm{dt}=\mathrm{s}_{2} \mathrm{~F}_{42}-\mathrm{s}_{4} \mathrm{~F}_{24}$

Considerando o coeficiente de diferencial de $\mathrm{s}_{3}$, quanto ao tempo, obteve-se:

$\mathrm{ds}_{3} / \mathrm{dt}=\mathrm{d}\left(\mathrm{q}_{3} / \mathrm{Q}_{3}\right) / \mathrm{dt}=\left[\mathrm{dq}_{3} / \mathrm{dt}-\left(\mathrm{q}_{3} / \mathrm{Q}_{3}\right) \mathrm{dQ} \mathrm{Q}_{3} / \mathrm{dt}\right] / \mathrm{Q}_{3}$

Reorganizando o sistema de equações, encontrou-se: $\mathrm{dq}_{3} / \mathrm{dt}=\mathrm{Q}_{3} \mathrm{ds}_{3} / \mathrm{dt}+\mathrm{s}_{3} \mathrm{dQ}_{3} / \mathrm{dt}$ e utilizando-se as equações (03) e (07) para substituir por $\mathrm{dQ}_{3} / \mathrm{dt}$ e $\mathrm{dq}_{3} / \mathrm{dt}$, respectivamente, e $\mathrm{ds}_{3} / \mathrm{dt}$ por $\left[\mathrm{s}_{3}(\mathrm{t}=\mathrm{t})-\mathrm{s}_{3}(\mathrm{t}=0)\right] /[\mathrm{t}-0]$, a equação (10) tornou-se:

$\mathrm{s}_{3} / \mathrm{t}=\left(\mathrm{s}_{2}-\mathrm{s}_{3}\right) \mathrm{F}_{32} / \mathrm{Q}_{3}$

Como s3 ( $\mathrm{t}=0$ ) é zero e considerado similar, ao aplicar para $\mathrm{ds}_{4} / \mathrm{dt}$, obteve-se:

$\mathrm{s}_{4} / \mathrm{t}=\left(\mathrm{s}_{2}-\mathrm{s}_{4}\right) \mathrm{F}_{42} / \mathrm{Q}_{4}$

Depois de oito dias de introdução do material radioativo, assumiu-se o pool 1 (lúmen do intestino) como em estado

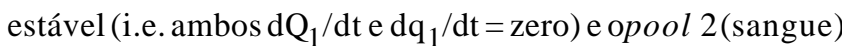
em estado não-isotópico estável (i.e. $\mathrm{dQ}_{2} / \mathrm{dt}=$ zero).

Assim, as equações (01), (02), (05), (11) e (12) foram transformadas:

$\mathrm{F}_{10}+\mathrm{F}_{12}-\mathrm{F}_{01}-\mathrm{F}_{21}=0$

$\mathrm{F}_{21}+\mathrm{F}_{23}+\mathrm{F}_{02}-\mathrm{F}_{12}-\mathrm{F}_{32}-\mathrm{F}_{42}=0$

$\mathrm{s}_{2} \mathrm{~F}_{12}-\mathrm{s}_{1}\left(\mathrm{~F}_{01}+\mathrm{F}_{21}\right)=0$

$\left(\mathrm{s}_{2}-\mathrm{s}_{3}\right) \mathrm{F}_{32} / \mathrm{Q}_{3}=\mathrm{s}_{3} / 8$

$\left(\mathrm{s}_{2}-\mathrm{s}_{4}\right) \mathrm{F}_{42} / \mathrm{Q}_{4}=\mathrm{s}_{4} / 8$

Pela manipulação algébrica das equações (13) a (17), obteve-se:

$\mathrm{F}_{12}=\mathrm{s}_{1} \mathrm{~F}_{10} /\left(\mathrm{s}_{2}-\mathrm{s}_{1}\right)$

$\mathrm{F}_{21}=\mathrm{F}_{10}+\mathrm{F}_{12}-\mathrm{F}_{01}$

$\mathrm{F}_{32}=\mathrm{s}_{3} \mathrm{Q}_{3} /\left[8\left(\mathrm{~s}_{2}-\mathrm{s}_{3}\right)\right]$

$\mathrm{F}_{42}=\mathrm{s}_{4} \mathrm{Q}_{4} /\left[8\left(\mathrm{~s}_{2}-\mathrm{s}_{4}\right)\right]$

$\left|\mathrm{F}_{23}+\mathrm{F}_{24}\right|=\mathrm{F}_{01}+\mathrm{F}_{12}+\mathrm{F}_{32}+\mathrm{F}_{42}-\mathrm{F}_{21}$

em que o $\left|\mathrm{F}_{23}+\mathrm{F}_{24}\right|$ indica um fluxo experimentalmente medido. $O$ fluxo combinado $\left|F_{23}+F_{24}\right|$ denota a soma dos fluxos de saída dos pools 3 e 4, equação 23, podendo ser separado pela combinação desses dois pools. $\mathrm{O}$ s* denota a atividade específica desse pool combinado, sendo calculado de acordo com a equação (24).

$\left|\mathrm{F}_{23}+\mathrm{F}_{24}\right|=\mathrm{F}_{23}+\mathrm{F}_{24}$

$\mathrm{s}^{*}=\left(\mathrm{s}_{3} \mathrm{Q}_{3}+\mathrm{s}_{4} \mathrm{Q}_{4}\right) /\left(\mathrm{Q}_{3}+\mathrm{Q}_{4}\right)$

O fluxo de saída do marcador a partir do pool combinado consistiu na soma do fluxo de saída do marcador dos pools 3 e 4 :

$\mathrm{s}^{*} \mathrm{x}\left|\mathrm{F}_{23}+\mathrm{F}_{24}\right|=\mathrm{s}_{3} \mathrm{~F}_{23}+\mathrm{s}_{4} \mathrm{~F}_{24}$

Por meio de manipulação algébrica das equações (23) e (25), obteve-se: 
$\mathrm{F}_{24}=\left(\mathrm{s}^{*}-\mathrm{s}_{3}\right) \mathrm{x}\left|\mathrm{F}_{23}+\mathrm{F}_{24}\right| /\left(\mathrm{s}_{4}-\mathrm{s} *\right)$

$\mathrm{F}_{23}=\left|\mathrm{F}_{23}+\mathrm{F}_{24}\right|-\mathrm{F}_{24}$

O modelo foi aplicado usando-se as equações (18) a (22), (24), e (26) a (27) para computar os fluxos conhecidos.

Obtidos os valores dos fluxos supracitados, o total de Ca endógeno nas fezes $\left(\mathrm{Fe}_{01}\right)$ foi calculado segundo a equação 28:

$\mathrm{F}_{\mathrm{e} 01}=\mathrm{F}_{12} \mathrm{~F}_{01} /\left(\mathrm{F}_{12}+\mathrm{F}_{10}\right)=\mathrm{s}_{1} \mathrm{~F}_{01} / \mathrm{s}_{2}$ em que $\mathrm{F}_{10}$ é o consumo de $\mathrm{Ca}$ e $\mathrm{F}_{01}$ o $\mathrm{Ca}$ nas fezes.

A absorção real de Ca (Fabs) foi calculada de acordo com a equação 29 :

$\mathrm{F}_{\mathrm{abs}}=\mathrm{F}_{10}-\left(\mathrm{F}_{01}-\mathrm{F}_{\mathrm{e} 01}\right)$

O modelo matemático 2 foi elaborado por Fernández (1995), ilustrado na Figura 2, e adaptado por Lopes et al. (2001), e representado pelas equações de 30 a 36 : $V_{a T}=V_{a a}+V_{a f}$,

$V_{f T}=V_{f} /\left[1-\left(V_{a a} / V_{I}\right)\right]$

$\mathrm{V}_{0+}=$ atividade acumulada nos ossos/integral da SRA no plasma

$V_{o-}=V_{o+}-B a$

$V_{a a}=-1.5+3.1 \ln V_{I}$

$V_{f}=V_{a a}-V_{I}+V_{F}$

$V_{a f}=V_{f T}-V_{f}$

Os fluxos correspondem a $F_{21}\left(\mathrm{~V}_{\mathrm{a}} \mathrm{T}\right), \mathrm{F}_{12}\left(\mathrm{~V}_{\mathrm{i}} \mathrm{T}\right), \mathrm{F}_{32}$ $\left(\mathrm{Vo}^{+}\right), \mathrm{F}_{23}\left(\mathrm{Vo}^{-}\right), \mathrm{F}_{42}\left(\mathrm{Vte}^{+}\right)$e $\mathrm{F}_{24}\left(\mathrm{Vte}^{-}\right)$.

A descrição das variáveis representadas na Figura 2 encontra-se na Tabela 2.

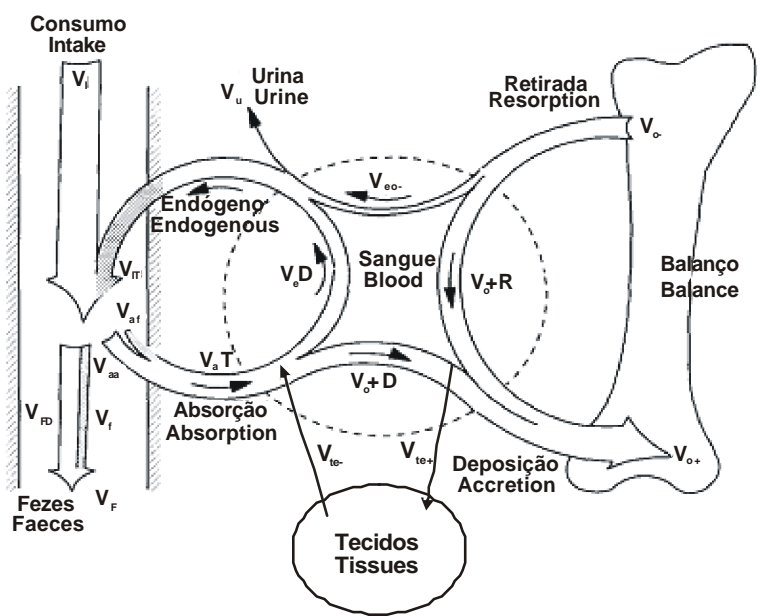

Figura 2 - Modelo da distribuição do Ca de acordo com Fernandez (1995).

Figure 2 - Metabolism of Ca according to Fernandez (1995) model.
Tabela 2 - Variáveis relacionadas ao metabolismo de $\mathrm{Ca}$, de acordo com modelo de Fernández (1995)

Table 2 - Variables for Ca metabolism proposed in the Fernandez (1995) model

\begin{tabular}{|c|c|}
\hline $\mathrm{V}_{\mathrm{I}}$ & Quantidade de Ca ingerido (Ca intake) \\
\hline$V_{F}$ & Perda total de Ca nas fezes (total fecal Ca loss) \\
\hline$V_{f}$ & $\mathrm{Ca}$ endógeno fecal (endogenous fecal $\mathrm{Ca}$ ) \\
\hline $\mathrm{V}_{\mathrm{FD}}$ & $\begin{array}{l}\text { Ca fecal de origem dietética }(\text { fecal } \mathrm{Ca} \\
\text { from dietary origin) }\end{array}$ \\
\hline $\mathrm{V}_{\mathrm{u}}$ & Perda de Ca na urina (urinary Ca loss) \\
\hline $\mathrm{V}_{\mathrm{aa}}$ & $\begin{array}{l}\text { Ca absorvido de origem alimentar (Ca absorbed } \\
\text { from dietary origin) }\end{array}$ \\
\hline $\mathrm{V}_{\mathrm{IT}}$ & $\begin{array}{l}\text { Ca de origem endógena que chega ao trato } \\
\text { gastrintestinal TGI (endogenous Ca that reaches } \\
\text { the gastrointestinal (GI) tract) }\end{array}$ \\
\hline $\mathrm{V}_{\mathrm{af}}$ & $\begin{array}{l}\text { Ca endógeno reabsorvido no TGI } \\
\text { Endogenous Ca reabsorbed in the GI tract }\end{array}$ \\
\hline $\begin{array}{l}\mathrm{V}_{\mathrm{aT}} \\
\mathrm{V}_{0+}\end{array}$ & $\begin{array}{l}\text { Total de Ca absorvido (total absorption of } \mathrm{Ca} \text { ) } \\
\mathrm{Ca} \text { incorporado no osso (bone Ca accretion) }\end{array}$ \\
\hline $\mathrm{V}_{0}^{-}$ & Ca reabsorvido do osso (bone Ca resorption) \\
\hline $\mathrm{V}_{\mathrm{eO}}^{-}$ & $\begin{array}{l}\text { Ca reabsorvido do osso e que retorna } \\
\text { ao TGI (reabsorbed bone Ca that is recycled } \\
\text { to the GI tract) }\end{array}$ \\
\hline $\mathrm{V}_{\mathrm{O}+\mathrm{D}}$ & $\begin{array}{l}\text { Ca do VaT incorporado no osso } \\
\text { VaT Ca deposited in the bone }\end{array}$ \\
\hline $\mathrm{V}_{\mathrm{te}+}$ & $\begin{array}{l}\mathrm{Ca} \text { incorporado nos tecidos moles } \\
\text { Ca deposited in the soft tissues }\end{array}$ \\
\hline $\mathrm{V}_{\mathrm{te}}{ }^{-}$ & $\begin{array}{l}\text { Ca reabsorvido dos tecidos moles } \\
\text { Reabsorbed Ca from soft tissues }\end{array}$ \\
\hline$V_{o+R}$ & $\begin{array}{l}\text { Total de Ca reciclado para o osso e os tecidos moles } \\
\text { Total Ca recycled into bone and soft tissues }\end{array}$ \\
\hline
\end{tabular}

\section{Resultados e Discussão}

Os dados do Ca consumido e excretado, das atividades específicas e da concentração de Ca nos ossos, no sangue e nos tecidos encontram-se na Tabela 3. Não houve diferença significativa no consumo de $\mathrm{Ca}\left(\mathrm{F}_{10}\right)$ entre as fontes de $\mathrm{Ca}$ e na quantidade de $\mathrm{Ca}$ na urina $\left(\mathrm{F}_{02}\right)$. $\mathrm{O}$ consumo de $\mathrm{Ca}$ foi adequado para todos os tratamentos e manteve-se acima de $0,51 \%$ da MS.

Embora o consumo tenha sido semelhante para todos os tratamentos, observou-se que a excreção fecal $\left(\mathrm{F}_{01}\right)$ foi menor para o tratamento calcário (CC) e correspondeu, em média, a 55\% do Ca consumido; para as demais fontes, o valor médio foi de $90 \%$. Os altos valores de Ca fecal podem indicar menor disponibilidade de $\mathrm{Ca}$ na fonte 
Tabela 3 - Consumo e excreção de cálcio, atividades específicas (ATE) e teores de cálcio no sangue, nas fezes, nos ossos e nos tecidos de ovinos em crescimento

Table 3 - Intake, excretion, specific radioactivity (SRA), and levels of calcium in blood, feces, bone and soft tissues of growing sheep

\begin{tabular}{|c|c|c|c|c|c|c|}
\hline \multirow[t]{3}{*}{$\begin{array}{l}\text { Item }^{1} \\
\text { Item }^{l}\end{array}$} & \multirow[t]{3}{*}{$\begin{array}{c}\text { Símbolo } \\
\text { Symbol }\end{array}$} & \multicolumn{5}{|c|}{$\begin{array}{c}\text { Fonte de } \mathrm{Ca} \\
\text { Ca source }\end{array}$} \\
\hline & & $\mathrm{CC}$ & FA & PC & FB & FC \\
\hline & & $L M$ & $A H$ & $C P$ & $D P$ & $S M$ \\
\hline
\end{tabular}

Fluxos $(\mathrm{g} \mathrm{Ca} / \mathrm{d})$

Flows

\begin{tabular}{lcccccc}
\hline $\begin{array}{l}\text { Consumo } \\
\text { Intake }\end{array}$ & $F_{10}$ & 5,20 & 6,20 & 6,33 & 6,77 & 6,26 \\
$\begin{array}{l}\text { Fezes } \\
\text { Feces }\end{array}$ & $F_{01}$ & $2,88^{\mathrm{b}}$ & $6,24^{\mathrm{a}}$ & $6,26^{\mathrm{a}}$ & $5,20^{\mathrm{a}}$ & $5,03^{\mathrm{ab}}$ \\
$\begin{array}{l}\text { Urina } \\
\text { Urine }\end{array}$ & $F_{02}$ & 0,06 & 0,04 & 0,12 & 0,15 & 0,08 \\
\hline
\end{tabular}

ATE $(\mathrm{com} / \mathrm{g} \mathrm{Ca})$

SRA

\begin{tabular}{lcccccc}
\hline $\begin{array}{l}\text { Fezes } \\
\text { Feces }\end{array}$ & $s_{1}$ & $19,67^{\mathrm{a}}$ & $6,00^{\mathrm{b}}$ & $8,00^{\mathrm{b}}$ & $8,33^{\mathrm{b}}$ & $8,00^{\mathrm{b}}$ \\
$\begin{array}{l}\text { Sangue } \\
\text { Blood }\end{array}$ & $s_{2}$ & 52,33 & 48,33 & 45,00 & 53,0 & 50,33 \\
$\begin{array}{l}\text { Osso } \\
\text { Bone }\end{array}$ & $s_{3}$ & $0,45^{\mathrm{ab}}$ & $0,37^{\mathrm{b}}$ & $0,34^{\mathrm{b}}$ & $0,30^{\mathrm{b}}$ & $0,55^{\mathrm{a}}$ \\
$\begin{array}{l}\text { Tecidos } \\
\text { Tissues }\end{array}$ & $s_{4}$ & 17,02 & 10,02 & 4,42 & 3,98 & 14,51 \\
\hline
\end{tabular}

Quantidade Ca (g)

Amount of $\mathrm{Ca}(\mathrm{g})$

\begin{tabular}{lcccccc}
\hline $\begin{array}{l}\text { Ca sangue } \\
\text { Blood Ca }\end{array}$ & $Q_{2}$ & 11,94 & 15,03 & 15,83 & 13,65 & 12,99 \\
$\begin{array}{l}\text { Ca osso } \\
\text { Bone Ca }\end{array}$ & $Q_{3}$ & 24,09 & 32,71 & 29,11 & 38,21 & 26,43 \\
$\begin{array}{l}\text { Ca tecidos } \\
\text { Tissues } \mathrm{Ca}\end{array}$ & $Q_{4}$ & 1,85 & 3,79 & 3,16 & 3,70 & 1,97 \\
\hline
\end{tabular}

${ }^{1}$ Médias seguidas de letras distintas na linha diferem pelo teste Duncan ( $P>0,05)$.

${ }^{1}$ Means followed by different letters within a row are different by Duncan test $(P<0.05)$.

estudada, o que ocasiona maior quantidade de Ca passando diretamente pelo trato digestivo, sem que haja absorção. No tratamento CC, além de menor excreção de Ca nas fezes, a atividade específica $\left(s_{1}\right)$, que representa a relação entre o Ca radioativo e a quantidade de Ca no trato gastrintestinal, também apresentou valor maior $(\mathrm{P}<0,05)$, indicando maior fluxo $\mathrm{F}_{12}$ ( $\mathrm{Ca}$ do sangue para trato gastrintestinal). A radioatividade nas fezes tem origem no compartimento central, representado pelo sangue, e consiste no Ca absorvido e utilizado pelo animal (Ca endógeno). Quando calculados os valores de Ca endógeno fecal e da absorção, obtiveram-se médias de 0,82 e 2,31; de 1,11 e 1,18; de 0,80 e 2,03; de 0,77 e 0,73; e de 1,08 e 3,40, respectivamente, para FB, PC, FC, FA e CC e, a partir desses valores, calculou-se a disponibilidade biológica $(34,12 ; 18,64 ; 32,43 ; 11,77$ e $65,38 \%$ para FB, PC, FC, FA e CC). Esses dados confirmam a afirmativa anterior de que o $\mathrm{Ca}$, na fonte $\mathrm{CC}$, apresentou valor maior de disponibilidade biológica.

A proporção entre a perda endógena fecal de $\mathrm{Ca}$ e o $\mathrm{Ca}$ consumido resultou, em média, em 12,11; 17,54; 12,77; 12,42 e 20,77\%, respectivamente para FB, PC, FC, FA e CC, o que está de acordo com os resultados descritos por Martz et al. (1990), que obtiveram para o Ca endógeno fecal valores de 16 a $20 \%$.

Pelos resultados obtidos, constatou-se que as fontes que apresentaram o Ca na forma orgânica (FA e PC) foram as que tiveram menores valores de absorção. De acordo com McDowell (1992), a forma química, o tipo de fonte e a interação com outros nutrientes afetam a absorção do mineral. Geralmente, a forma orgânica é a menos disponível.

No caso da dieta PC, um dos fatores que podem ter afetado a absorção foi a presença de pectina, que, na polpa cítrica, pode ser em torno de 19\% (Giraldo, 1999). A pectina liga-se a cátions e pode prejudicar a absorção de minerais como Ca, Mg, Cu e Zn. O balanço negativo observado para FA pode ter sido ocasionado pela presença de oxalato, composto presente nas fontes orgânicas de Ca que diminui a disponibilidade de $\mathrm{Ca}$. No feno de alfafa, uma parte do $\mathrm{Ca}(20$ a $30 \%)$ está presente na forma de oxalatos insolúveis de $\mathrm{Ca}$ e aparentemente não disponíveis ao animal (Ward et al., 1979).

As quantidades de $\mathrm{Ca}$ no sangue, nos ossos e nos tecidos moles não diferiram entre si $(\mathrm{P}>0,05)$. No entanto, a fonte calcário calcítico apresentou menor valor absoluto da concentração desse mineral nas variáveis estudadas, o que, provavelmente, implicou maior fluxo de $\mathrm{Ca}$ do tecido para o sangue e deste para o trato digestivo.

Constam na Tabela 4 os fluxos de entrada e saída de Ca nos compartimentos, considerando os diversos tratamentos.

No tratamento CC, os fluxos entre o trato gastrintestinal e o sangue $\left(F_{21}\right.$ e $\left.F_{12}\right)$, em ambas as direções, foram maiores $(\mathrm{P}<0,05)$. A diferença entre entrada e saída de $\mathrm{Ca}\left(\mathrm{F}_{21}-\mathrm{F}_{12}\right)$ resultou na quantidade absorvida, que apresentou valores de 2,$33 ;-0,05 ; 0,06 ; 1,56$ e $1,23 \mathrm{~g} \mathrm{Ca} /$ dia, respectivamente, para CC, FA, PC, FB e FC.

Pela relação entre $\mathrm{Ca}$ absorvido e $\mathrm{Ca}$ consumido, obteve-se 0,$45 ;-0,01 ; 0,01 ; 0,23$ e 0,20 , respectivamente, para CC, FA, $\mathrm{PC}, \mathrm{FB}$ e FC, portanto, valores mais elevados para CC, FB e FC. Esses dados confirmam os achados anteriores de que as fontes $\mathrm{CC}, \mathrm{FB}$ e FC apresentam maior disponibilidade de Ca (Bao et al., 1997)

Os fluxos sangue-ossos $\left(\mathrm{F}_{32}\right)$ e sangue-tecidos moles $\left(F_{42}\right)$ (Tabela 4) foram mais elevados $(\mathrm{P}<0,05)$ para o tratamento CC. No caso do $F_{32}$, verificou-se valor do fluxo do CC foi similar ao das fontes FC e FB. Quanto aos fluxos $\mathrm{F}_{23} \mathrm{e}$ 
Tabela 4 - Média dos fluxos de Ca em ovinos para as diferentes fontes de Ca de acordo com os modelos matemáticos de Vitti e Fernandez Table 4 - Means for Ca flows in sheep fed different Ca sources according to Vitti et al. (2000) and Fernandez (1995) mathematical models

\begin{tabular}{|c|c|c|c|c|c|c|}
\hline \multirow[t]{2}{*}{$\begin{array}{l}\text { Item }^{1} \\
\text { Item }\end{array}$} & \multirow[t]{2}{*}{$\begin{array}{c}\text { Símbolo } \\
\text { Symbol }\end{array}$} & \multicolumn{5}{|c|}{$\begin{array}{c}\text { Fonte de } \mathrm{Ca} \\
\text { Calcium source }\end{array}$} \\
\hline & & $\mathrm{CC}$ & FA & $\mathrm{PC}$ & FB & $\mathrm{FC}$ \\
\hline \multicolumn{7}{|l|}{ Fluxos (g/dia) } \\
\hline Sangue para osso (From blood to bone) & $F_{32}\left(\mathrm{~V}_{\mathrm{o}+}^{11}\right)$ & $1,10^{\mathrm{a}}$ & $0,04^{\mathrm{b}}$ & $0,61^{\mathrm{b}}$ & $0,85^{\mathrm{a}}$ & $0,63^{\mathrm{a}}$ \\
\hline Osso para sangue (From bone to blood) & $F_{23}^{32}\left(\mathrm{~V}_{\mathrm{o}-}^{\mathrm{o}}\right)$ & $0,01^{\mathrm{a}}$ & $0,11^{\mathrm{a}}$ & $0,17^{\mathrm{a}}$ & $0,01^{\mathrm{a}}$ & $0,01^{\mathrm{a}}$ \\
\hline Sangue para tecidos (From blood to tissues) & $F_{42}\left(\mathrm{~V}_{\text {tet }}\right)$ & $1,17^{\mathrm{a}}$ & $0,14^{\mathrm{b}}$ & $0,10^{\mathrm{b}}$ & $0,57^{b}$ & $0,52^{b}$ \\
\hline Tecidos para sangue (From tissues to blood) & $F_{24}\left(\mathrm{~V}_{\mathrm{te}-}\right)$ & $0,01^{\mathrm{a}}$ & $0,14^{\mathrm{a}}$ & $0,11^{\mathrm{a}}$ & $0,01^{\mathrm{a}}$ & $0,01^{\mathrm{a}}$ \\
\hline
\end{tabular}

${ }_{1}^{1}$ Médias seguidas de letras distintas na linha diferem $(P>0,05)$ pelo teste Duncan.

${ }^{1}$ Means followed by different letters within a row differ $(P<0.05)$ by Duncan test.

Tabela 5 - Fluxos de Ca obtidos pelos modelos de Vitti e Fernandez para ovinos recebendo diferentes fontes de cálcio Table 5 - Flows of Ca according to the Vitti et al. (2000) and Fernandez (1995) models in sheep fed diets with different Ca sources

\begin{tabular}{|c|c|c|c|c|c|c|}
\hline \multirow[t]{2}{*}{$\begin{array}{l}\text { Item }^{1} \\
\text { Item }\end{array}$} & \multirow[t]{2}{*}{$\begin{array}{l}\text { Símbolo } \\
\text { Symbol }\end{array}$} & \multicolumn{5}{|c|}{$\begin{array}{c}\text { Fonte de } \mathrm{Ca} \\
\text { Calcium source }\end{array}$} \\
\hline & & CC & FA & PC & FB & $\mathrm{FC}$ \\
\hline
\end{tabular}

Dados modelo Vitti (g P/d)

Vitti et al. (2000) model data

\begin{tabular}{|c|c|c|c|c|c|c|}
\hline Trato para sangue (From gut to blood) & $F_{21}$ & $5,51^{\mathrm{a}}$ & $0,90^{\mathrm{b}}$ & $1,50^{\mathrm{b}}$ & $2,82^{\mathrm{ab}}$ & $2,49^{\mathrm{b}}$ \\
\hline Sangue para trato (From blood to gut) & $F_{12}^{21}$ & $3,18^{\mathrm{a}}$ & $0,94^{\mathrm{b}}$ & $1,49^{\mathrm{ab}}$ & $1,25^{\mathrm{b}}$ & $1,26^{\mathrm{b}}$ \\
\hline Sangue para osso (From blood to bone) & $F_{32}$ & $1,85^{\mathrm{a}}$ & $0,03^{\mathrm{c}}$ & $1,14^{\mathrm{c}}$ & $1,26^{\mathrm{ab}}$ & $0,98^{\mathrm{b}}$ \\
\hline Osso para sangue (From bone to blood) & $F_{23}^{32}$ & $0,01^{\mathrm{a}}$ & $0,16^{\mathrm{a}}$ & $0,24^{\mathrm{a}}$ & $0,01^{\mathrm{a}}$ & $0,01^{\mathrm{a}}$ \\
\hline Tecidos para sangue (From tissues to blood) & $F_{24}$ & $0,01^{\mathrm{a}}$ & $0,02^{\mathrm{a}}$ & $0,03^{\mathrm{a}}$ & $0,01^{\mathrm{a}}$ & $0,01^{\mathrm{a}}$ \\
\hline Retenção líquida nos ossos (Net bone retention) & $F_{32}-F_{23}$ & 1,85 & $-0,13$ & 0,90 & 1,26 & 0,98 \\
\hline Retenção líquida nos tecidos (Net soft tissues retention) & $F_{42}^{32}-F_{24}^{23}$ & 0,41 & 0,05 & 0,03 & 0,15 & 0,18 \\
\hline
\end{tabular}

Dados modelo Fernández (g P/d)

Fernández (1995) model data

\begin{tabular}{|c|c|c|c|c|c|c|}
\hline Trato para sangue (From gut to blood) & $V_{a T}$ & $5,40^{\mathrm{a}}$ & $0,85^{\mathrm{b}}$ & $1,60^{\mathrm{b}}$ & $2,77^{b}$ & $2,46^{\mathrm{b}}$ \\
\hline Sangue para trato (From blood to gut) & $V_{I T}$ & $3,08^{\mathrm{a}}$ & $0,89^{\mathrm{b}}$ & $1,53^{\mathrm{b}}$ & $1,20^{\mathrm{b}}$ & $1,23^{\mathrm{b}}$ \\
\hline Osso para sangue (From bone to blood) & $V_{o-}^{o+}$ & $0,01^{\mathrm{a}}$ & $0,05^{\mathrm{a}}$ & $0,09^{\mathrm{a}}$ & $0,01^{\mathrm{a}}$ & $0,01^{\mathrm{a}}$ \\
\hline Tecidos para sangue (From tissues to blood) & $V t e^{-}$ & $0,01^{\mathrm{b}}$ & $0,26^{\mathrm{a}}$ & $0,19^{\mathrm{ab}}$ & $0,01^{\mathrm{b}}$ & $0,01^{\mathrm{b}}$ \\
\hline Retenção líquida nos ossos (Net bone retention) & $V_{o+}-V_{o-}$ & 0,35 & $-0,01$ & $-0,01$ & 0,43 & 0,28 \\
\hline Retenção líquida nos tecidos (Net tissues retention) & $V t e^{+}-V_{t}^{-}$ & 1,91 & $-0,06$ & $-0,06$ & 0,99 & 0,85 \\
\hline
\end{tabular}

${ }_{1}^{1}$ Médias seguidas de letras distintas na linha diferem $(P>0,05)$ pelo teste Duncan.

${ }^{1}$ Means followed by different letters within a row differ $(P<0.05)$ by Duncan test.

$\mathrm{F}_{24}$, os valores foram semelhantes para todas as fontes estudadas $(\mathrm{P}>0,05)$.

Na Tabela 5 são ilustrados os valores dos fluxos obtidos pelos dois modelos individualmente. Esses dados apresentaram as mesmas tendências dos descritos na Tabela 4, ou seja, os valores foram maiores para as entradas e saídas entre trato e sangue no tratamento CC. Em ambos os modelos, o balanço de $\mathrm{Ca}$ nos ossos e tecidos foi maior para CC. O balanço de $\mathrm{Ca}$ nos ossos foi negativo para o FA em ambos os modelos e a retenção nos tecidos foi negativa para FA e PC no modelo Fernandez.

$\mathrm{O}$ balanço positivo indicou deposição de Ca tanto nos ossos como nos tecidos moles. Nos tratamentos nos quais o balanço foi negativo, ocorreu mobilização de $\mathrm{Ca}$ dos ossos e tecidos para manter os níveis sangüíneos adequados ao desempenho das funções vitais no animal.

A comparação entre os modelos de Vitti et al. (2000) e Fernandez (1995) é representada na Tabela 6. 
Tabela 6 - Comparação dos fluxos de Ca para os modelos de Vitti e Fernandez utilizando-se os dados obtidos em todos os tratamentos

Table 6 - Comparison of Ca flows using Vitti et al. (2000) and Fernandez (1995) models for all treatments

\begin{tabular}{|c|c|c|c|c|c|c|}
\hline \multirow[t]{2}{*}{$\begin{array}{l}\text { Modelo } \\
\text { Model }\end{array}$} & \multicolumn{6}{|c|}{$\begin{array}{c}\text { Fluxo de Ca entre os compartimentos } \\
\text { Flow of Ca between compartments }\end{array}$} \\
\hline & $\mathrm{F}_{12}$ & $\mathrm{~F}_{21}$ & $\mathrm{~F}_{32}$ & $\mathrm{~F}_{23}$ & $\mathrm{~F}_{42}$ & $\mathrm{~F}_{24}$ \\
\hline Vitti et al. (2000) & $1,62^{\mathrm{a}}$ & $2,65^{\mathrm{a}}$ & $0,85^{\mathrm{a}}$ & $0,08^{\mathrm{a}}$ & $0,14^{\mathrm{b}}$ & $0,01^{\mathrm{b}}$ \\
\hline Fernandez (1995) & $2,38^{\mathrm{a}}$ & $2,62^{\mathrm{a}}$ & $0,24^{\mathrm{b}}$ & $0,03^{\mathrm{b}}$ & $0,82^{\mathrm{a}}$ & $0,09^{\mathrm{a}}$ \\
\hline
\end{tabular}

Houve semelhança para os fluxos entre o sangue e o trato gastrintestinal em ambos os sentidos, para os dois modelos. Os modelos diferiram nos fluxos que envolvem ossos e tecidos moles. No modelo Vitti, os fluxos de Ca entre osso e sangue foram maiores $(\mathrm{P}<0,01)$ que no modelo Fernandez e os fluxos entre tecidos e sangue apresentaram-se mais elevados pelo modelo de Fernandez $(\mathrm{P}<0,01)$. Entretanto, nos balanços finais (Tabela 5), tanto para ossos como para tecidos, houve similaridade entre os valores obtidos, que foram maiores para $\mathrm{CC}$ e $\mathrm{FB}$.

As diferenças entre os dois modelos possivelmente foram ocasionadas pela estrutura desses modelos. No modelo Vitti, utilizaram-se medidas diretas de atividades específicas (análises químicas e contagens radioativas), incluídas na maioria das equações para cálculo dos fluxos, estando, portanto, sujeito a erros experimentais. O modelo de Fernandez, no entanto, foi desenvolvido de modo menos estruturado e mais empírico, não utilizando tantas medidas experimentais diretas.

\section{Conclusões}

O calcário calcítico, o fosfato bicálcico e a farinha de conchas de ostras apresentaram maior disponibilidade de cálcio $(65,38 ; 34,12$ e $32,43 \%$, respectivamente), enquanto, nas fontes orgânicas polpa cítrica e feno de alfafa, verificou-se menor disponibilidade (16,46 e $11,77 \%$, respectivamente) desse mineral para o organismo de ovinos da raça Santa Inês.

O fluxo do cálcio do sangue para os ossos e para os tecidos moles foi mais elevado para o calcário calcítico e, portanto, houve maior retenção.

O fluxo do cálcio dos ossos e dos tecidos moles para o sangue foi similar para calcário calcítico, fosfato bicálcio, farinha de conchas de ostras, polpa cítrica e feno de alfafa.

$\mathrm{Na}$ fonte $\mathrm{FA}$, o balanço de $\mathrm{Ca}$ nos ossos foi negativo, indicando maior mobilização do mineral para o sangue.
Os modelos matemáticos aplicados para o metabolismo de $\mathrm{Ca}$ em ovinos concordam na maioria dos dados de entrada e saída dos compartimentos e ambos indicam que as fontes inorgânicas proporcionam melhor aproveitamento de Ca pelos animais.

\section{Literatura Citada}

ANDRIGUETTO, J.M.; PERLY, L.; MINARDI, I. Nutrição animal. São Paulo: Nobel, 1993. 395p.

AUBERT, J.P.; MILHAUD, G. Méthod de measure des principales voies du métabolisme calcique chez l'homme. Biochimica et Biophysica Acta, v.39, n.1, p.122-139, 1960.

BAO, S.F.; WINDISCH, W.; KIRCHGESSNER, M. Calcium availability of different organic and inorganic dietary Ca sources. Journal of Physiology and Animal Nutrition, v.78, p. 154160,1997

BECK, A.B.; BUGEL, S.; JENSEN, M. et al. A novel dual radio- and stable- isotope method for measuring calcium absorption in humans: comparison with the whole - body radioisotope retention method. American Journal of Clinical Nutrition, v.377, p.399-405, 2003.

BOSTON, R.C.; GREIF, P.C.; BERMAN, M. Conversational SAAMan interactive program for kinetics analysis of biological systems. Computer Programs in Biomedicine, v.13, p.111119,1981 .

BRAVO, D.; SUVANT, D.; BOGAERT, C. et al. A bibliographic database for quantitative analysis of phosphorus flow in ruminants. Reproduction, Nutrition, and Development, v.43, p.251-269, 2003.

CHALLA, J.; BRAITHWAITE, G.D.; DHANOA, M.S. Phosphorus homeostasis in growing calves. Journal of Agricultural Science, v.112, n.2, p.217-226, 1989.

FERNANDEZ, J.A. Calcium and phosphorus metabolism in growing pigs. III. A model resolution. Livestock Production Science, V.41, n.1, p.255-261, 1995.

GIRALDO, M.A.M. Estratégias para a avaliação nutricional da polpa cítrica seca em suínos em terminação. Belo Horizonte: Universidade Federal de Minas Gerais, 1999. 95p. Tese (Doutorado em Zootecnia) - Universidade Federal de Minas Gerais

GRACE, N.D. Phosphorus kinetics in the sheep.British Journal of Nutrition, v.45, p.367-374, 1981 .

KEBREAB, E.; MILLS J.; CROMPTON L.A. et al. An integrated mathematical model to evaluate nutrient partition in dairy cattle between animal and environment. Animal Feed Science and Technology, v.112, p.131-154, 2004

LOPES, J.B.; VITTI, D.M.S.S.; ABDALLA, A.L. et al. Modelo do fluxo do fósforo de fontes de fosfato em suínos, usando-se $\mathrm{o}^{32} \mathrm{P}$ como marcador. Revista Brasileira de Zootecnia, v.30, n.1, p.165-173, 2001 .

MARTZ, F.A.; BELO, A.T.; WEISS, M.F. et al. True absorption of calcium and phosphorus from alfalfa and corn silage when fed to lactating cows. Journal of Dairy Science, v.73, n.5, p.1288-1295, 1990.

McDOWELL, L.R. Minerals in animal and human nutrition San Diego: Academic Press, 1992. 524p.

MEJÍA, A.M.G.; FERREIRA, W.M. Produção e caracterização bromatológica da polpa cítrica seca. Revista CFMV, v.19, p.23-33, 2000. (Suplemento técnico).

MORSE, D.; HEAD, H.H.; WILLOX, C.J. et al. Effects of concentration of Dietary Phosphorus on amount and route of excretion. Journal of Dairy Science, v.75, n.11, p.30393049, 1992.

NATIONAL RESEARCH COUNCIL - NRC. Nutrient requirement of sheep. Washington, D.C.: National Academic Press, 1985.99p. 
PEIXOTO, R.R.; MAIER, J.C. Nutrição e alimentação animal. 2.ed. Pelotas: UCPel; EDUCAT; UFPel, 1993. 169p.

PFEFFER. E.; BEEDE, D.K.; VALK, H. Phosphorus metabolism in ruminants and requirements of cattle. In: PFEFFER, E.; HRISTOV, A. (Eds.) Nitrogen and phosphorus nutrition of cattle. Wallingford: CABI Publishing, 2005. p.195-231.

SALVIANO, L.M.C.; VITTI, D.M.S.S. Influência da proporção de cálcio e fósforo na dieta, nas perdas endógenas e na absorção de fósforo em ovinos. Pesquisa Agropecuária Brasileira, v.33, n.3, p.349-355, 1998.

STATISTICAL ANALYSIS SYSTEM - SAS. The SAS system for windows. Release 8.01. Cary: 2000. (CR-ROM).

SCHNEIDER, K.N.; TERNOUTH, J.H.; SEVILLA, C.C. et al. A short-term study of calcium and phosphorus absorption in sheep fed on diets high and low in calcium and phosphorus. Australian Journal Agricultural Research, v.36, p.91-105. 1985.

VITTI, D.M.S.S.; ABDALLA, A.L.; MEIRELLES, C.F. et al. Absorção real do fósforo de diferentes fontes para ovinos através do uso de radiofósforo. Pesquisa Agropecuária Brasileira, v.27, n.5, p.1405-1408, 1992.

VITTI, D.M.S.S.; KEBREAB, E.; LOPES, J.B. et al. A kinetic model of phosphorus metabolism in growing goats. Journal of Animal Science, v.78, p.2706-2712, 2000.
VITTI, D.M.S.S.; KEBREAB, E.; LOPES, J.B. et al. Effects of dietary supplementation on phosphorus metabolism in sheep. Journal of Animal and Veterinary Advances, v.4, p.349355. 2005.

WARD, G.; HARBERS, L.H.; BLAHA, J.J. Calcium containing crystals in alfalfa: their fate in cattle. Journal of Dairy Science, v.62, n.5, p.715-722, 1979.

ZAGATTO, E.A.G.; KRUG, F.J.; BERGAMIN FILHO, H. et al. Merging zones in flow injection analysis. Part II. Determination of calcium, magnesium and potassium in plant material by flow injection atomic absorption and flame emission spectrometry. Analytical Chemistry, v.104, p.279-284, 1979.

Recebido: 12/12/05 Aprovado: 14/06/06 